\title{
Combination of Sleeve Gastrectomy and Mesenchymal Stem Cells Improves Insulin Resistance by Modulating Pro-inflammatory Milieu in Obese Type 2 Diabetic Rat Model
}

Vito Mahendra Ekasaputra ${ }^{1,2} \mathbb{D}$, Abdul Mughni $^{3} \mathbb{D}$, Agung Putra $^{4,5,6 *} \mathbb{D}$, Ignatius Riwanto ${ }^{3}$, Dimas Erlangga Nugrahadi ${ }^{3}$,

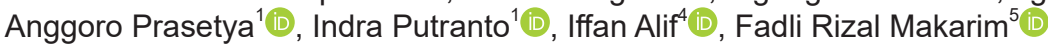

${ }^{1}$ Department of Surgery, Faculty of Medicine, Diponegoro University, Kariadi Hospital, Semarang, Indonesia; ${ }^{2}$ Department of Surgery, Faculty of Medicine, Sultan Agung Islamic University, Semarang, Indonesia; ${ }^{3}$ Department of Digestive Surgery, Faculty of Medicine, Diponegoro University, Kariadi Hospital, Semarang, Indonesia; ${ }^{4}$ Stem Cell and Cancer Research, Faculty of Medicine, Sultan Agung Islamic University, Semarang, Indonesia; ${ }^{5}$ Department of Pathological Anatomy, Faculty of Medicine, Sultan Agung Islamic University, Semarang, Indonesia; ${ }^{6}$ Department of Postgraduate Biomedical Science, Faculty of Medicine, Sultan Agung Islamic University, Semarang, Indonesia

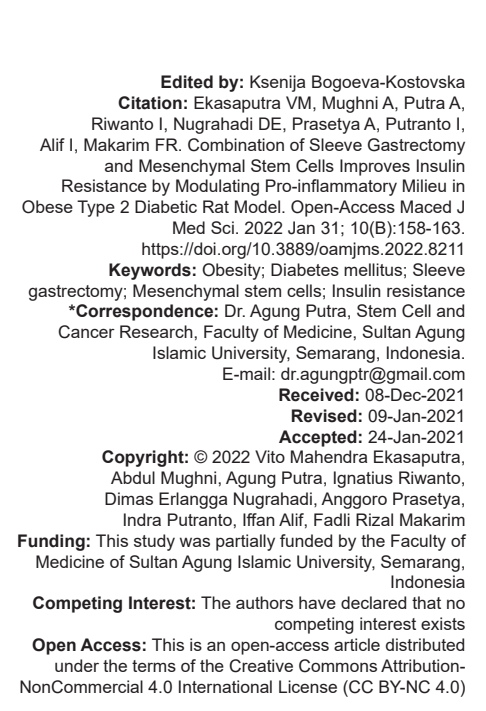

\section{Abstract}

BACKGROUND: Obesity is a major risk factor for the onset of insulin resistance (IR) and type 2 diabetes mellitus (T2DM) caused by chronic inflammation of the islets of Langerhans. Sleeve gastrectomy (SG) procedure increases particular hormone which stimulates insulin sensitivity. Mesenchymal stem cells (MSCs) can also exhibit potential immunomodulatory properties through their paracrine effects; however, the mechanism regarding combination of them could not be adequately explained.

AIM: In this study, we explore the potential of SG followed by injection of MSCs in type 2 diabetic rats with obesity in improving IR.

MATERIALS AND METHODS: This study used a pre and post control group design with 18 rats that divided into three groups: Control (C), SG, and SG + MSCs (SG+M). On day 10, the level of interleukin (IL-6), IL-10, and homeostasis model assessment of IR (HOMA-IR) was evaluated using ELISA.

RESULTS: This study showed a significant decrease of IL-6 level in all treatment groups on day 10, in which SG $+M$ group showed optimum inhibition. This result was in line with the optimum increase of IL-10 in SG + M group. Moreover, our study also revealed the optimum decrease of HOMA-IR in SG + M group on day 10.

CONCLUSION: Combination of SG and MSCs can optimally improve IR by modulating pro-inflammatory milieu though inhibiting IL-6 level and upregulating IL-10 level in obese T2DM rat model.

\section{Introduction}

Obesity is a major risk factor for the onset of insulin resistance (IR), hyperinsulinemia, and type 2 diabetes mellitus (T2DM) that are associated with high rate of morbidity and mortality [1], [2]. It is well known that weight loss is associated with decrease of IR [3], [4]; however, management of weight loss based on diet, exercise, and behavioral therapy are associated with poor long-term results [2]. The previous studies reported that bariatric surgery offers an important and sustained weight loss along with amelioration/remission of T2DM [1], [3], [4]. Moreover, rapid improvement of IR and early resolution of T2DM have been observed to occur after bariatric surgery [3], implying that these changes are unrelated to weight loss and more likely induced by some hormonal effects of the bariatric procedures through the enteroinsular axis. Bariatric surgery can also remediate inflammation, which is majority caused by the increase level of interleukin (IL6). This inflammatory inhibition tending to be greatest in those with greatest weight loss, however, the patients experienced delayed improvement of insulin sensitivity with prolonged secretion of pro-inflammatory cytokines [3], [4].

Sleeve gastrectomy (SG) is a pure restrictive operation and has been initially described as a modification of biliopancreatic diversion and as part of a staged surgical approach for high- risk morbidly obese patients. Moreover, recently, it has been used as a sole weight loss operation. SG is regarded as a 
surgical intervention that has a more simple restrictive procedure, which could significantly reduce fat mass, however, yet the precise mechanisms involved in the early control of blood glucose after SG are still unclear [4], [5].

Mesenchymal stem cells (MSCs) are multipotent stem cells with self-renewing capacities and low immunogenicity, which make them attractive for treating many diseases. MSCs naturally express various surface markers such as CD73, CD90, CD105, and lack the expression of CD45, CD34, CD14 or CD11b, and CD79a or CD19. Under standard in vitro differentiation conditions, MSCs can differentiate into specific cells such as chondrocytes, osteocytes, and adipocytes. Interestingly, a recent paradigm shift suggests that MSCs have exhibited potential immunomodulation and anti-inflammatory properties through their paracrine effects [6]. MSCs can downregulate the pro-inflammatory milieu through expressing anti-inflammatory molecules, such as IL-10, transforming growth factor- $\beta$, and prostaglandin E2 [7]. Moreover, the previous studies have shown that MSCs can reduce systemic inflammation in patients with T2DM and alleviates IR in target tissues of T2D rats. Given the beneficial anti-inflammatory property of MSCs, it is imperative to study the possibility of MSCs in suppressing inflammatory milieu to improve IR [7], [8].

In this study, we performed SG followed by intraperitoneal injection of MSCs in obese diabetic rats. We investigated inflammatory milieu and IR level through analyzing the IL-6, IL-10, and homeostasis model assessment of IR (HOMA-IR).

\section{Materials and Methods}

\section{Experimental animals}

Eighteen male Wistar rats weighing 170-200 g were purchased from the Laboratorium Penelitian dan Pengembangan Terpadu, Universitas Gadjah Mada, Yogyakarta, Indonesia. Rats were caged at $24 \pm 2{ }^{\circ} \mathrm{C}$ and $60 \%$ relative humidity, with $12: 12 \mathrm{~h}$ light-dark cycle (laboratory standard). All the animals were humanely treated in accordance with the guidelines for animal care and the protocols were approved by the Ethics Committee of Health Studies, Faculty of Medicine, Universitas Diponegoro (permit number: 51/EC/H/ FK-UNDIP/V/2021).

\section{Induction of obese diabetic rats}

After 1 week adaptation period, the rats were randomly divided into three groups: Control group (C), SG only (SG), and SG followed with 1 cc site intraperitoneal injection containing $1 \times 10^{6}$ of MSCs
$(S G+M)$. Rats were fed based on high caloric and fatty diet, consist of $60 \%$ Comfeed pars, $27.8 \%$ starch, $2 \%$ cholesterol, $0.2 \%$ cholic acid, $10 \%$ lard, and 2 cc fructose/day. After 4 weeks, rats were injected with $230 \mathrm{mg} / \mathrm{kg}$ nicotinamide (NA) intraperitoneally $15 \mathrm{~min}$ before single dose of intraperitoneal administration of $65 \mathrm{mg} / \mathrm{kg}$ streptozotocin (STZ) to induce a diabetic animal model.

\section{SG}

Seven days after STZ induction, the abdominal cavity rats on $S G$ and $S G+M$ groups were opened with an oblique left subcostal incision under general anesthesia using $20 \mathrm{mg} / \mathrm{kg}$ body weight of ketamine hydrochloride. The stomach was dissected above the mayor curvature line until it remained $50 \%$ of its volume. The residual stomach was then closed using continuous suture of 5-0 PDS ${ }^{\circledR}$. Moreover, abdominal wall was closed with whole layer simple suture 3-0 Vicryl (Figure 1).

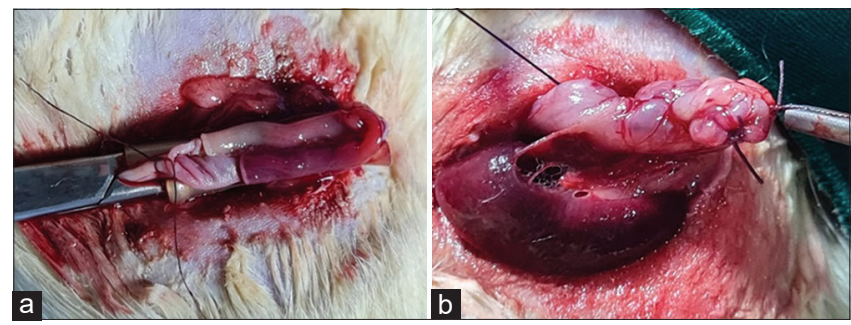

Figure 1: Sleeve gastrectomy procedure in T2DM obese rat. (a) Modified clamp with rubber protection within residual part of stomach to reduce the bleeding. (b) Suture of residual stomach

\section{Isolation and culture of MSCs}

MSCs were isolated from human umbilical cords. Phosphate buffer solution (PBS) (Gibco TM Invitrogen, NY, USA) with 5\% Penstrep antibiotic was used as a transport medium. The Wharton's jelly was separated from umbilical cord and minced evenly then placed into the $75 \mathrm{~cm}^{2}$ flask containing Dulbecco's Modified Eagle's Medium (DMEM) (Sigma-Aldrich, Louis St., MO) mixed with $10 \%$ fetal bovine serum (FBS) (Gibco) TM Invitrogen, NY, USA) and $100 \mathrm{lU} / \mathrm{mL}$ penicillin/streptomycin (Sigma-Aldrich). The cultured Wharton's Jelly was incubated in $5 \% \mathrm{CO}_{2}$ and $37^{\circ} \mathrm{C}$ incubator. The medium was changed in 3 days intervals and the MSCs will emerge in 7-10 days. After reaching $80 \%$ confluence, the MSCs were passaged by trypsin. The $4^{\text {th }}$ passage was used for the experiment. This study was approved by the Institutional Review Board of the Ethics Committee of the Medical Department, Sultan Agung Islamic University, Semarang, Indonesia [8].

\section{Characterization of MSCs}

To confirm the MSC-like surface antigens, plastic adherent stromal cells at the fourth passage 
were characterized by flow cytometry assays. After trypsinized and pelleted, the cells were subsequently incubated using fluorescein allophycocyanin (APC)-, isothiocyanate (FITC)-, phycoerythrin (PE)-, and peridinin-chlorophyll-protein (perCP)-Cy5.5.1conjugated anti-rat CD90.1, CD29, CD31, and CD45 antibodies (BD Bioscience, San Jose, CA, USA) for $30 \mathrm{~min}$ at room temperature in the dark. On the other hand, an isotype-specific conjugated anti-lgG (BD Bioscience, San Jose, CA, USA) was used as negative controls. The cells were then washed twice using PBS. The analyses were performed using a BD Accuri C6 Plus flow cytometer (BD Bioscience, San Jose, CA, USA). The post-acquisition analysis was performed using the BD Accuri C6 Plus software (BD Bioscience, San Jose, CA, USA).

\section{Osteogenic and Adipogenic Differentiation of MSCs}

We further performed the osteogenic differentiation assay in the fifth passage. The MSC-like cells were cultured in a standard medium containing DMEM with $10 \%$ FBS, $1 \%$ penicillin (100 U/mL)/ streptomycin $(100 \mu \mathrm{g} / \mathrm{mL})$, and $0.25 \%$ amphotericin $\mathrm{B}$ at $37^{\circ} \mathrm{C}$ and $5 \% \mathrm{CO}_{2}$ until reaching $95 \%$ confluency. Then, the standard medium was replaced using an osteogenic differentiation medium containing Rat MesenCult ${ }^{\mathrm{TM}}$ Osteogenic Differentiation Basal Medium (Stem Cell Technologies, Singapore) with 20\% Rat MesenCult ${ }^{\mathrm{TM}}$ Osteogenic Differentiation 5X Supplement (Stem Cell Technologies, Singapore), 1\% L-glutamine (Gibco ${ }^{\mathrm{TM}}$ Invitrogen, NY, USA), 1\% penicillin $(100 \mathrm{U} / \mathrm{mL}) /$ streptomycin $(100 \mu \mathrm{g} / \mathrm{mL})$, and $0.25 \%$ amphotericin B. On the other side, for inducing adipogenic differentiation, the UC-MSClike with $95 \%$ confluency were cultured using adipogenic differentiation medium which made of Rat MesenCult ${ }^{\mathrm{TM}}$ MSC Basal Medium (Stem Cell Technologies), Rat MesenCult ${ }^{\mathrm{TM}}$ Adipogenic Differentiation Supplement (Stem Cell Technologies), 1\% L-Glutamine (Gibco ${ }^{\mathrm{TM}}$ Invitrogen), 1\% penicillin/ streptomycin (100 U/mL; respectively) (Gibco $^{\mathrm{TM}}$ Invitrogen), and $0.25 \%$ amphotericin B $(62.5 \mu \mathrm{g} /$ $\mathrm{mL}$ ) (Gibco ${ }^{\mathrm{TM}}$ Invitrogen). The medium was changed every 3 days. Calcium and lipid deposition were shown by alizarin red staining and oil red $\mathrm{O}$ staining, respectively (Sigma-Aldrich, Louis St, MO), followed by 21 days of induction.

\section{MSCs administration}

After SG procedure, the SG + M group was treated by MSCs intraperitoneally at doses of $1 \times 10^{6}$. On the other hand, the control and the SG groups were intraperitoneally received natrium chloride $(\mathrm{NaCl})$.

\section{Blood glucose monitoring}

Seven days after induction of STZ and 10 days after SG and MSCs injection, blood sample was taken from infraorbital venous of rats. Fasting plasma glucose levels were determined by blood samples using the Accu-Chek (Roche, Basel, Switzerland).

\section{IL-6, IL-10, insulin, and HOMA-IR level analysis}

The serum from blood samples at day 10 was separated and analyzed by ELISA kit for measuring IL-6, IL-10, insulin, and HOMA-IR. The assay was performed at room temperature according to the manufacturer's instructions (Sigma-Aldrich). The results were analyzed at a wavelength of $450 \AA$ using a microplate reader (Bio-Rad, CA, USA). Specifically, HOMA-IR was used to evaluate IR before and after treatment using the following formula HOMA-IR= (fasting insulin $\mu \mathrm{U} / \mathrm{ml} \times$ fasting glucose $\mathrm{mg} / \mathrm{dl} / \mathrm{/} / 22.5 \times 18$.

\section{Statistical analysis}

The statistical analysis was performed using SPSS ver. 23 (SPSS Inc., Chicago, IL, USA). Data were produced by mean \pm standard deviation (SD). For intergroup comparison, the one-way ANOVA followed by post hoc test using Tukey's post hoc was used. $p<0.05$ was considered statistically significant.

\section{Results}

\section{Human MSCs (H-MSCs) characterization and differentiation}

The MSCs were isolated from rat umbilical cord under standard culture condition for five passages. The cells showed fibroblast-like with spindle shape characteristic (Figure 2a). The osteogenic and adipogenic differentiation was identified by administrating osteogenic and adipogenic differentiation medium for 21 days. After Alizarin red and oil red $O$ staining, calcium and adipose deposition was appeared on H-MSCs in red color indicated the multipotency (Figure $2 \mathrm{~b}$ and $\mathrm{c}$ ). The flow cytometric analysis showed high level of CD90.1 (99.4\%), CD29 (96.9\%) lacked the expression of CD45 (1.9\%) and CD31 (3.7\%) (Figure 2d).

\section{Combination of SG and MSCs optimally downregulates IL6 level in obese diabetic rats}

To investigate the capacity of SG and its combination with MSCs in suppressing proinflammatory cytokine, the level of IL-6 was measured 


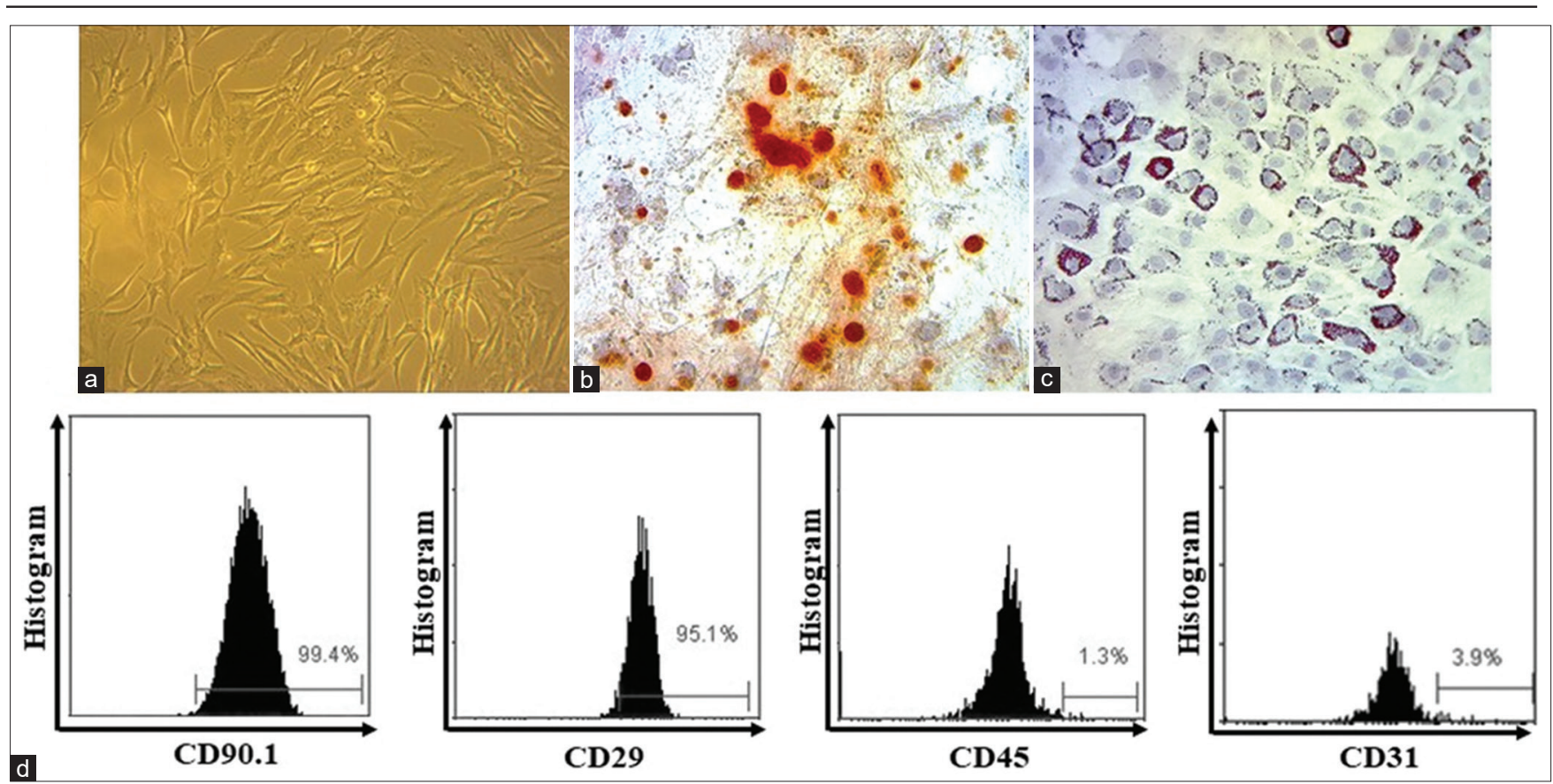

Figure 2: (a) In vitro culture of H-MSC showed spindle-like shaped characteristic (magnification 100x); (b) Osteogenic and (c) adipogenic differentiation of H-MSCs was evidenced by calcium and adipose deposition appearance as a matrix visualized by red color using Alizarin red and oil red o, respectively (scale bar $50 \mu \mathrm{m}$ ) (magnification 100x); (d) Immunophenotyping characterization of H-MSCs showed high level of $C D 90.1, C D 29$, and low expression of $C D 45, C D 31$

using ELISA 10 days after treatments. ELISA assay showed that there was a significant decrease of IL-6 level in all treatment groups, compared to control group $(p<0.05)$. The optimum decrease of IL6 was shown in $\mathrm{SG}+\mathrm{M}$ group with $77.56 \pm 2.526 \mathrm{pg} / \mathrm{mL}$ (Figure 3).

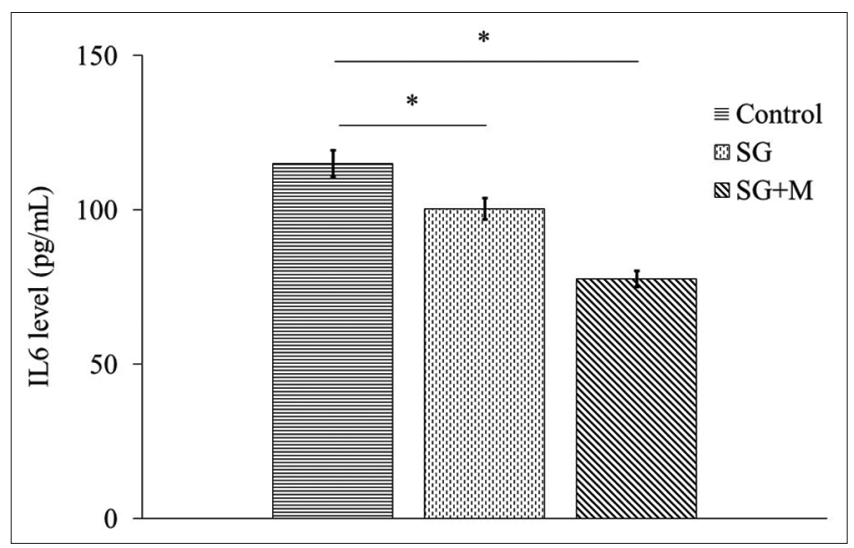

Figure 3: Suppressive capacity of sleeve gastrectomy and its combination with mesenchymal stem cells on interleukin-6 level in type 2 diabetes mellitus rats. ${ }^{*} p<0.05$. Data are expressed as means $\pm S D$

\section{Combination of SG and MSCs optimally} enhances IL10 level in obese diabetic rats

We next examined the capacity of SG and its combination with MSCs in enhancing anti-inflammatory cytokine. The level of IL-10 was measured using ELISA 10 days after treatments. As shown in Figure 4, the level of IL-10 was significantly increased in all treatment groups, compared to control $(p<0.05)$. The $S G+M$ group showed optimum enhancement of IL10 level with $80.30 \pm 2.971 \mathrm{pg} / \mathrm{mL}$.

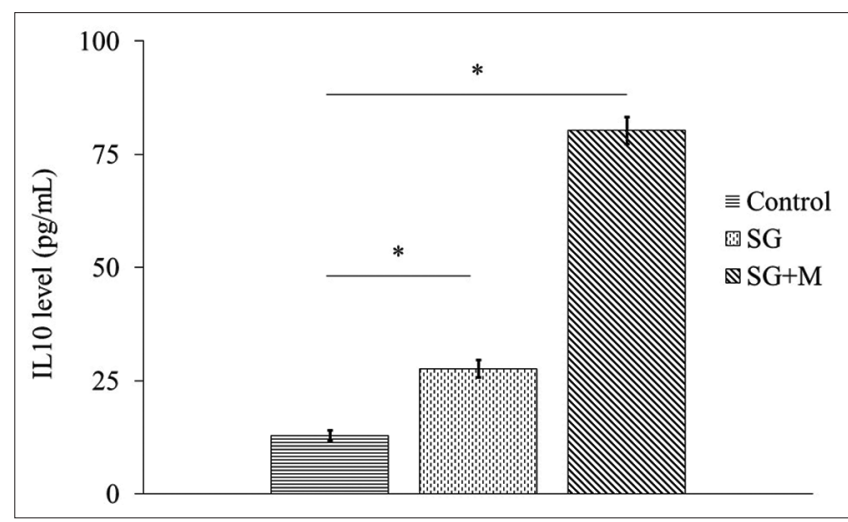

Figure 4: Sleeve gastrectomy and its combination of mesenchymal stem cells enhance the level of interleukin 10 in type 2 diabetes mellitus rats. ${ }^{*} p<0.05$. Data are expressed as means $\pm S D$

\section{Combination of SG and MSCs optimally improves IR in obese diabetic rats}

To examine the capacity of SG and its combination in improving IR, we analyzed the level of HOMA-IR measured using ELISA. Specifically, HOMA-IR was analyzed before and after treatment using the following formula HOMA-IR = (fasting insulin $\mu \mathrm{U} / \mathrm{ml} \times$ fasting glucose $\mathrm{mg} / \mathrm{dl} / / / 22.5 \times 18$. First, we analyzed the level of HOMA-IR before treatments. ELISA assay showed that there was no significant difference of HOMA-IR level between all groups $(p>0.05)$. Ten days after treatments, we measured the level of HOMA-IR and we found that there was significant decrease in treatment groups, compared to control. The optimum decrease of HOMA-IR was shown in SG + M group with $49.77 \pm$ 1.07 (Figure 4). 


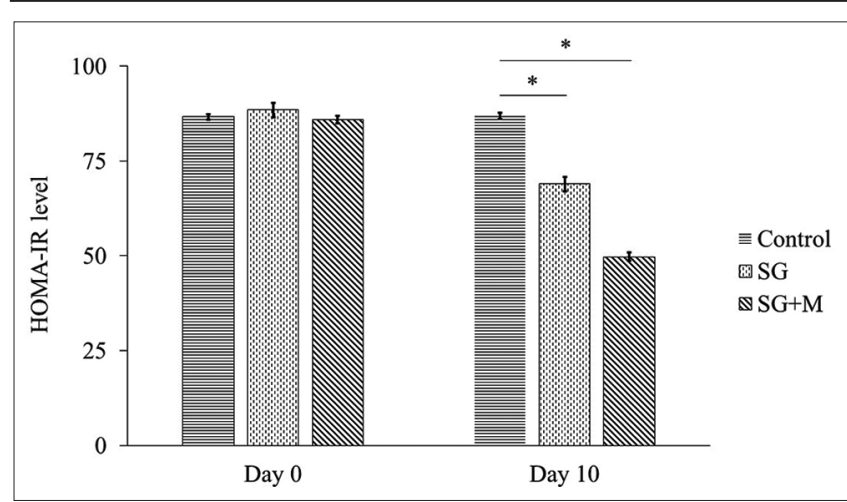

Figure 5: Sleeve gastrectomy and its combination of mesenchymal stem cells decrease the level of HOMA-IR in type 2 diabetes mellitus rats. ${ }^{*} p<0.05$. Data are expressed as means $\pm S D$

\section{Discussion}

The release of pro-inflammatory cytokines, such as IL6 and TNF $\alpha$, has been indicated to cause IR, and anti-inflammatory therapeutic strategy is becoming promising in the treatment of obese T2DM. The previous study showed that bariatric surgery, such as SG, offers an important and sustained weight loss along with significant amelioration of T2DM [1], [3], [4]. SG could reduce anti-inflammatory cytokine through decreasing of adipocyte tissue, these results proved that ameliorating of IR was influenced by inflammatory cytokines [4]. Moreover, rapid improvement of IR and early resolution of T2DM have been observed to occur after bariatric surgery due to lower inflammation cytokine secretes by adipocyte tissue [9]. Consistent with prior study, we also observed MSCs administration that not only improved IR but also impaired the pro-inflammatory milieu, marked by reduced caspase-1 activity, and decreased expression of IL1 $\beta$, IL18, IL6, and TNF $\alpha$ [10], [11]. Studies also showed that MSCs effectively promoted insulin action by stimulating the insulin receptor signaling pathway in target tissues [12]. These studies guided us to further explore the potential of SG and MSCs to provide future clinical therapeutic approaches of obese T2DM.

This study showed that SG could potentially inhibit pro-inflammatory mediators, including IL-6 through enhancing IL-10 and improving HOMA-IR in T2DM. The previous study reported that hormonal mechanisms are involved to improve T2DM condition. Ghrelin that mainly produced by the gastric fundus could play a role in suppressing the insulin-sensitizing hormone adiponectin, block hepatic insulin signaling, and inhibit insulin secretion. Thus, the removal of the gastric fundus through SG may lead to reduced ghrelin secretion and therefore improving the glucose metabolism. Other studies also revealed that $Y Y$ peptide (PYY) and glucagon-like peptide 1 (GLP-1) were increased after SG. A potential explanation for the increase of PYY and GLP- 1 following LSG is the accelerated gastric emptying and earlier contact of chyme with the L cells of the hindgut [5], [13].
Our study also showed that the combination of SG and MSCs provides excessive inhibition of IL6 level and robust enhancement of IL-10 level. These data suggest that MSCs may impede the pro-inflammatory niche under T2DM conditions. At a sufficient proinflammatory condition, MSCs can upregulate the expression of TLR-3 and PGE2, leading to the activation of TRIF-TRAM-mediated anti-inflammatory signals and the release of various anti-inflammatory molecules, including IL10 [14]. IL10 as a potent antiinflammatory cytokine could inhibit the overexpression of pro-inflammatory mediators, including IL6 in T2DM. Specifically, excessive level of IL10 could promote the activation of suppressor of cytokine signaling 3 (SOSC3), leading to the inhibition of nuclear factor kappa B (NF$\mathrm{kB}$ ) and signal transducer and activator of transcription 3 (STAT3). This mechanism could attenuate the expression of several pro-inflammatory cytokines, including IL-6 [15]

On the other hand, this study demonstrated that the combination of SG and MSCs offers major improvement in IR. The previous studies reported that insulin binds to its receptor to trigger a series of insulin signaling transduction pathways. The IRS-PI3K-Akt pathway plays an important role in insulin's metabolic effects. Ser-307 phosphorylation of IRS-1 is considered an important negative indicator of IR followed by reduced PI3K/Akt phosphorylation. PI3K Akt phosphorylates and further increases the Glut4 protein content in insulin target tissues. The translocations of Glut4 to cell membranes of target tissues are responsible for improvement in sensitivity to insulin action [16]. Proinflammatory mediators, such as IL6, TNF $\alpha$, and IFN $\gamma$, are the major factor in promoting IR. These molecules could activate NFkB and inhibit the expression of IRS-1, leading to the decrease expression of Glut4 [17]. MSCs could robustly suppress the expression of pro-inflammatory mediators so that enhancing the insulin-stimulated IRSPI3K-Akt pathway, leading to the recovery of IR [18]. These results suggest the concept that the anti-inflammatory action of MSCs is responsible for improving IR in target tissues of $T 2 D$ rats.

There are several limitations in this study, the animal model used here may not reflect the true diabetes mellitus pathogenesis of IR cause by obesity. Long-term outcome was also not evaluated to assess recurrence of diabetes after bariatric surgery. We also did not analyze the intracellular molecules involved in inflammatory condition, such as SOSC3, NF-kB, STAT3, and insulin pathway such as IRS, PI3K, and Akt. Further experimental studies were needed to evaluate the whole combination procedure before clinical study.

\section{Conclusion}

This study demonstrated that in obese diabetic rat, combination of SG and MSCs can optimally improve 
IR. This improvement is followed by the decrease level of IL6 as a potent pro-inflammatory cytokine and the enhance level of IL10 as a major anti-inflammatory cytokine, simultaneously. Our findings are important for understanding the potency of SG and its combination with MSCs as a potent immunomodulatory mediator in obese T2DM.

\section{Acknowledgment}

This research was partial funded by Faculty of Medicine of Sultan Agung Islamic University Semarang, Indonesia. We would also like to thank to Stem Cell and Cancer Research (SCCR) Laboratory, Medical Faculty, Sultan Agung Islamic University (Unissula), Semarang, for all facility to finish this study.

\section{References}

1. Morton JM. The ASMBS Textbook of Bariatric Surgery. 2nd ed. Springer; 2020.

2. McArdle MA, Finucane OM, Connaughton RM, McMorrow AM, Roche HM. Mechanisms of obesity-induced inflammation and insulin resistance: Insights into the emerging role of nutritional strategies. Front Endocrinol (Lausanne). 2013;4:1-23. https:// doi.org/10.3389/fendo.2013.00052

PMid:23675368

3. Nicolas W. Cortes-Penfield, Barbara W. Trautner RJ. Orexin activation counteracts decreases in nonexercise activity thermogenesis (NEAT) caused by high-fat diet. Physiol Behav. 2017;176(5):139-48. https://doi.org/10.1016/j. physbeh.2017.03.040

PMid:28363838

4. Biobaku F, Ghanim H, Monte SV, Caruana JA, Dandona P. Bariatric surgery: Remission of inflammation, cardiometabolic benefits, and common adverse effects. J Endocr Soc. 2020;4(9):bvaa049.

PMid:32775937

5. Kassem MA, Durda MA, Stoicea N, Cavus O, Sahin L, Rogers B. The impact of bariatric surgery on Type 2 diabetes mellitus and the management of hypoglycemic events. Front Endocrinol (Lausanne). 2017;8:37. https://doi.org/10.3389/ fendo.2017.00037

PMid:28298900

6. Putra A, Rosdiana I, Darlan DM, Alif I, Hayuningtyas F, Wijaya I, et al. Intravenous administration is the best route of mesenchymal stem cells migration in improving liver function enzyme of acute liver failure. Folia Med (Plovdiv). 2020;62(1):52-8. https://doi. org/10.3897/folmed.e47712

PMid:32337897

7. Putra A, Sa'diyah NA, Rahmalita A, et al. The effect of mesenchymal stem cells on the endothelial cells of diabetic mice. In: Hofstra R, Koibuchi N, Fucharoen S, editors. Proceeding of $4^{\text {th }}$ BIBMC and $2^{\text {nd }}$ ACMM. Vol. 4. Boca Raton, Florida: CRC Press; 2017. p. 57-60.

8. Pawitan JA, Yang Z, Wu YN, Lee EH. Towards standardized stem cell therapy in Type 2 diabetes mellitus: A systematic review. Curr Stem Cell Res Ther. 2018;13(6):476-88.

PMid:29732994

9. Burhans MS, Hagman DK, Kuzma JN, Schmidt KA, Kratz M. Contribution of adipose tissue inflammation to the development of Type 2 diabetes mellitus. Compr Physiol. 2018;9(1):1-58. https://doi.org/10.1002/cphy.c170040

PMid:30549014

10. Muhar AM, Putra A, Warli SM, Munir D. Hypoxia-mesenchymal stem cells inhibit intra-peritoneal adhesions formation by upregulation of the il-10 expression. Open Access Maced J Med Sci. 2019;7(23):3937-43. https://doi.org/10.3889/ oamjms.2019.713

PMid:32165932

11. Muhar AM, Putra A, Ekasaputra VM, Darlan DM, Paramita DA, Adeputra Nasution IP. Mesenchymal stem cells under hypoxia condition inhibit peritoneal adhesion by suppressing the prolonged release of interleukin-6. Med Glas (Zenica). 2021;18(2):398-403. https://doi.org/10.17392/1304-21 PMid:34212709

12. Wang $M$, Song L, Strange $C$, Dong $X$, Wang $H$. Therapeutic effects of adipose stem cells from diabetic mice for the treatment of Type 2 diabetes. Mol Ther. 2018;26(8):1921-30. https://doi. org/10.1016/j.ymthe.2018.06.013

PMid:30005867

13. Abdalla MM. Ghrelin physiological functions and regulation. Eur Endocrinol. 2015;11(2):90-5. https://doi.org/10.17925/ EE.2015.11.02.90 PMid:29632576

14. Darlan DM, Munir D, Jusuf NK, Putra A, Ikhsan R, Alif I. In vitro regulation of IL- 6 and TGF- $\beta$ by mesenchymal stem cells in systemic lupus erythematosus patients. Med Glas (Zenica). 2020;17(2):408-13. https://doi.org/10.17392/1186-20 PMid:32602296

15. Niemand C, Nimmesgern A, Haan S, Fischer P, Schaper F, Rossaint R, et al. Activation of STAT3 by IL- 6 and IL-10 in primary human macrophages is differentially modulated by suppressor of cytokine signaling 3. J Immunol. 2003;170(6):3263-72. https://doi.org/10.4049/jimmunol.170.6.3263

PMid: 12626585

16. Copps KD, White MF. Regulation of insulin sensitivity by serine/ threonine phosphorylation of insulin receptor substrate proteins IRS1 and IRS2. Diabetologia. 2012;55(10):2565-82. https://doi. org/10.1007/s00125-012-2644-8 PMid:22869320

17. Chen L, Chen R, Wang H, Liang F. mechanisms linking inflammation to insulin resistance. Int $\mathrm{J}$ Endocrinol. 2015;2015:508409. https://doi.org/10.1155/2015/508409 PMid:26136779

18. Kotikalapudi N, Sampath SJ, Narayan SS, Bhonde R, Nemani H, Mungamuri SK, et al. The promise(s) of mesenchymal stem cell therapy in averting preclinical diabetes: Lessons from in vivo and in vitro model systems. Sci Rep. 2021;11(1):16983. https:// doi.org/10.1038/s41598-021-96121-0

PMid:34417511 